\title{
NONHEREDITARY SEMISIMPLE CLASSES
}

W. G. LEAVITT AND E. P. ARMENDARIZ

1. Introduction. For a class $\mathfrak{T}$ of rings let $\mathscr{g}_{1} \mathfrak{T} \ell=\{K \mid K$ is an ideal of some $R \in \mathscr{T}\}$ be called the ideal class of $\mathscr{M}$, and $\mathfrak{F C T}=\{K \mid K$ is a homomorphic image of some $R \in \mathfrak{M}\}$ the homomorphic closure of $\mathbb{M}$. A class $\mathfrak{T}$ is hereditary if $\mathfrak{g}_{1} \mathfrak{T C}=\mathfrak{M}$ and homomorphically closed if $\mathfrak{F} \mathfrak{T}=\mathfrak{T}$. A class which is both hereditary and homomorphically closed will be called a universal class. We will choose an arbitrary, but fixed, universal class $\mathcal{C}$ of (not necessarily associative) rings and assume that all rings considered are members of $\mathbb{e}$.

Note that, for $\mathfrak{N}$ an arbitrary class, $\mathcal{F} \mathfrak{T}$ is homomorphically closed and is minimal in the sense that if $\mathfrak{M} \subseteq \mathfrak{N}$ where $\mathfrak{N}$ is homomorphically closed, then $\mathfrak{F} \mathscr{T} \subseteq \mathfrak{T}$.

To construct a minimal hereditary class containing $\mathfrak{T}$, assume for induction that for a given integer $n \geqq 2$, classes $\mathscr{I}_{j} \mathscr{M}$ have been defined for all $j<n$. Then define $\mathfrak{I}_{n} \mathfrak{M}=\mathfrak{I}_{1} \mathfrak{I}_{n-1} \mathfrak{T}$. By induction $\mathfrak{I}_{n} \mathfrak{T}$ exists for all $n$. Call $\mathscr{g} \mathfrak{T}=U \mathcal{U}_{n} \mathfrak{T}$ the hereditary closure of $\mathfrak{M}$. It is clear by its definition that $\mathscr{g} \mathfrak{M}$ is hereditary and, by induction, any hereditary class containing $\mathfrak{M}$ also contains $\mathfrak{g} \mathscr{T}$.

It is well known that if $\rho$ is a radical class [1], [2] then

$$
S \odot=\left\{R \in \mathfrak{C} \mid \mathscr{I}_{1} R \cap \odot=0\right\}
$$

is a semisimple class $[3$, p. 3]. (Note: we write $R$ for the class $\{R\}$ containing $R$ as its sole member.) On the other hand, if $\mathcal{Q}$ is a semisimple class [3, Theorem 2, p. 5] then

$$
\mathcal{U Q}=\{R \in \mathcal{C} \mid \mathfrak{H C} R \cap \mathcal{Q}=0\}
$$

is a radical class $[3$, p. 6$]$, called the $u$ pper radical class defined by $\mathcal{Q}$. Note that the proofs of [3, Chapter I] apply equally well to nonassociative rings.

We will also use the Kurosh construction (modified by Anderson, Divinsky and Sulinsky [3, Footnote, p. 12]) of the lower radical defined for a class $\mathfrak{N}$ : Let $\mathfrak{N}_{1}=\mathfrak{K} \mathfrak{K}$ and assume that for a given ordinal $\beta$, classes $\mathfrak{T}_{\alpha}$ have been defined for all ordinals $\alpha<\beta$. Then define $\mathfrak{M}_{\beta}=\left\{R \in \mathcal{C} \mid\right.$ every nonzero $K \in \mathfrak{F} R$ satisfies the condition $\mathfrak{I}_{1} K \cap \Re_{\alpha}$ $\neq 0$ for some $\alpha<\beta\}$. Then the lower radical $\mathscr{L}=U_{\mathcal{B}}$.

2. Condition that a semisimple class be nonhereditary. It is well known that if $\mathfrak{e}$ is an associative class or an alternative class then

Received by the editors September 1, 1966. 
every semisimple class is hereditary [4, Corollary 2, p. 597 and Corollary 2, p. 602]. The question has been raised as to whether or not there exists a nonhereditary semisimple class. The following theorem gives a necessary and sufficient condition for the existence of such a class:

TheOREM 1. A universal class $\mathcal{C}$ contains a nonhereditary semisimple class if and only if there exists a ring $R \in \mathcal{C}$ such that $\mathfrak{I}_{1} R \cap \mathfrak{L}=0$ for some nonzero ring $J \in g R$.

Proof. From the definition (1) it follows that $g_{1} R \cap \mathscr{L} J=0$ implies $R \in \mathcal{L} J$. But for any radical $\mathscr{L} J \cap S \mathcal{L} J=0$, so since $J \in \mathscr{L} J$ it follows that $J \notin \mathfrak{S} \mathcal{L} J$. However $J \in \mathfrak{g} R$ whence $\mathscr{S} J$ is not hereditary.

Conversely, suppose the semisimple class $\mathfrak{N}$ is nonhereditary. Then there exists a ring $R \in \mathscr{T}$ such that $K \in \mathscr{T}$ for some $K \in \mathfrak{I}_{1} R$. By [3, Theorem 2, p. 5] there exists some $J \in \mathfrak{g}_{1} K$ such that $J \in \mathcal{U}$. By the minimality of the lower radical [3, Lemma 5, p. 13] this implies $\mathfrak{L} \subseteq \mathcal{U T}$. By the definition (2) of the upper radical, $น \mathfrak{T} \cap g_{1} \mathfrak{T}=0$. But $R \in \mathscr{M}$ whence $g_{1} R \subseteq g_{1} \mathscr{T}$ and so $g_{1} R \cap \mathscr{L} J=0$.

3. Construction of nonhereditary semisimple classes. In this section rings will be constructed satisifying Theorem 1 , with $J$ having contrasting properties; namely in one case $J^{2}=J$, and in the other case $J^{2}=0$. The class $\mathcal{C}$ in which these are to be constructed is the class of all (not necessarily associative) rings.

We will need to use specializations of the following lemma due to A. E. Hoffman [5]. For completeness the proof is supplied.

Lemma 1. Let $\mathfrak{N}$ be a hereditary class. If a class $\mathfrak{T}$ satisfies the condition $\mathfrak{H} \mathfrak{M} \cap \mathscr{N}=0$ then $\mathfrak{L} \cap \cap \Re=0$.

Proof. $\mathfrak{M}_{1}=\mathscr{K} \mathscr{N}$ satisfies $\mathfrak{M}_{1} \cap \mathscr{N}=0$ and assume for induction that for a given ordinal $\beta, \mathfrak{M}_{\alpha} \cap \Re=0$ for all ordinals $\alpha<\beta$. If there exists a nonzero $R \in \mathscr{N}_{\beta} \cap \mathscr{N}$, then by definition $\mathscr{G}_{1} R \cap \mathfrak{M}_{\alpha} \neq 0$ for some $\alpha<\beta$. But $g_{1} \mathfrak{N}=\mathscr{N}$ and since $R \in \mathfrak{N}$ it follows that $g_{1} R \subseteq \mathscr{N}$, contradicting the induction hypothesis. By transfinite induction $\mathfrak{N}_{\beta} \cap \Re=0$ for all ordinals $\beta$ and hence $\mathfrak{L} \cap \cap \mathscr{H}=0$.

A ring $K$ will be called hereditarily idempotent if $H^{2}=H$ for all $H \in \mathfrak{g} K$. A ring $R$ with the property $R^{2}=0$ will be called a zero ring. It is clear that we can specialize $\mathscr{N}$ in Lemma 1 to be either: (a) the class $D$ of all hereditarily idempotent rings, or (b) the class $\mathrm{Z}$ of all zero rings.

Consider the ring $K$ generated by nonassociative symbols $\{e, x, y\}$ over the field $Z_{2}$ of integers modulo 2, subject to the relations: 
$e^{2}=e x=x e=e, e y=y e=x y=y x=x, x^{2}=y^{2}=0$. It is clear that the only proper ideal of $K$ is $I=K^{2}=\{0, e, x, e+x\}$, and that $I$ contains the ideal $J=\{0, e\}$. Since $J^{2}=J$ and $J$ is simple, we have $Z \cap \mathfrak{H} J=0$. Thus by Lemma $1, Z \cap \mathfrak{L} J=0$. But if any ring $R \neq R^{2}$ then $R \notin \mathscr{L} J$ for otherwise the radical class $\& J$ would contain the zero ring $R / R^{2}$. Since the only nonzero ideals of $K$ are $K$, for which $I=K^{2} \neq K$, and $I$ for which $J=I^{2} \neq I$, it follows that $\mathfrak{g}_{1} K \cap \mathfrak{L} J=0$. Thus by Theorem 1 , $\mathcal{L} J$ is a nonhereditary class.

Using this example, we can prove:

TheOREM 2. The class 3 of all idempotent rings is a radical class whose semisimple class $\mathrm{SJ}$ is nonhereditary.

Proof. If $K \in J$ then $K^{2}=K$ so that $(K \phi)^{2}=K \phi$ for all homomorphisms $\phi$. Thus $J$ is homomorphically closed. On the other hand, $K \notin J$ implies $K^{2} \neq K$ so that $K / K^{2} \in \mathrm{Z}$. Thus $g_{1}\left(K / K^{2}\right) \subseteq g_{1} Z=Z$. Since $Z \cap J=0$, it follows that $g_{1}\left(K / K^{2}\right) \cap J=0$, that is $K / K^{2} \in \delta J$. By $[3$, Theorem 1$]$ J is a radical class. Now the ring $K$ constructed above has no idempotent ideals, so $K \in \mathcal{S} J$. But $J \in \mathfrak{g} K$ with $J \in \mathfrak{J}$ so, by Theorem $1, \delta J$ is nonhereditary.

Now consider the ring $R$ generated over $Z_{2}$ by the symbols $\{u, v, w\}$ satisfying relations: $u^{2}=0, u v=v u=u, \quad u w=w u=v w=w v=v^{2}=v$, $w^{2}=w$. It is clear that $I=\{0, u, v, u+v\}$ is the only proper ideal of $R$ and that $J=\{0, u\}$ is an ideal of $I$. Since $J^{2}=0$ and $J$ is simple, we have $\mathscr{D} \cap \mathfrak{H} J=0$. Thus by Lemma $1, \mathfrak{D} \cap \mathscr{L} J=0$. But $R / I, I / J \in \mathscr{D}$ so that $g_{1} R \cap \mathscr{L} J=0$. It follows from Theorem 1 that $\delta \mathscr{L} J$ is nonhereditary.

From this construction follows:

THEOREM 3. If $B$ is the lower Baer radical class (that is, $B=\mathcal{L Z}$ where $\mathrm{Z}$ is the class of all zero rings), then $\mathrm{S} B$ is a nonhereditary semisimple class.

Proof. In the ring $R$ constructed above $J \in \mathcal{Z}$ so that $\& J \subseteq \& Z=ß$. Now $Z$ is homomorphically closed and $D \cap Z=0$ so by Lemma 1 , $D \cap \mathscr{Z}=0$. Since every ideal of $R$ has an image in $D$, it follows that $R \in S B$. But $J \in B$ so $S B$ is nonhereditary.

Another consequence of this construction is: The class $\beta$ of all prime rings is nonhereditary. This follows from the fact that the ring $R$ just constructed is prime, whereas $J$ is not.

\section{REFERENCES}

1. A. G. Kurosh, Radicals of rings and algebras, Mat. Sb. 33 (1953), 13-26.

2. S. A. Amitsur, A general theory of radicals. II: Radicals in rings and bicategories, Amer. J. Math. 76 (1954), 100-125. 
3. N. J. Divinsky, Rings and radicals, Univ. of Toronto Press, Toronto, 1965.

4. T. Anderson, N. Divinsky and A. Sulinski, Hereditary radicals in associative and alternative rings, Canad. J. Math. 17 (1965), 594-603.

5. A. E. Hoffman, The constructions of the general theory of radicals, Ph.D. Thesis, University of Nebraska, Lincoln, 1966.

UNIVERSITY OF NEBRASKa AND

University of Southern California

\section{ON COHOMOLOGICAL TRIVIALITY}

\section{H. ONISHI}

Let $G$ be a finite group and $A$ be a $G$-module. It is well known that if for some two consecutive dimensions the cohomology groups $H^{r}(H, A)$ are trivial for all subgroups $H$ of $G$, then $H^{r}(H, A)$ are trivial for all dimensions $r$ and for all subgroups $H$ of $G$. This note is to point out the following generalization.

Theorem. Let $G$ be a finite group and $A$ be a G-module. If for some integer $k$ and for some odd positive integer $d, H^{k}(H, A)$ and $H^{k+d}(H, A)$ are trivial for all subgroups $H$ of $G$, then $H^{r}(H, A)$ are trivial for all integers $r$ and for all subgroups $H$ of $G$.

Proof. As usual we proceed by induction on the order $n=|G|$. The theorem is trivial for $n=1$. Suppose $n>1$ and assume the truth of the theorem for all groups of order $<n$. In particular, we may assume that $H^{r}(H, A)=0$ for every dimension $r$ and for every proper subgroup $H$ of $G$. If $n$ is not a prime power, then every Sylow subgroup of $G$ is a proper subgroup and the conclusion follows by a wellknown result.

Suppose now $n$ is a prime power, so that $G$ is solvable. Let $H$ be a proper normal subgroup of $G$ such that the quotient group $G / H$ is cyclic. Since $H^{r}(H, A)=0$ for every $r$, we have the Fundamental Exact Sequence of Hochschild and Serre [2];

$$
0 \rightarrow H^{r}\left(G / H, A^{H}\right) \rightarrow H^{r}(G, A) \rightarrow H^{r}(H, A)
$$

for every $r>0$. Since the last term is trivial, this says that $H^{r}\left(G / H, A^{\boldsymbol{H}}\right)$ $\cong H^{r}(G, A)$ for every $r>0$. By dimension shifting we could have assumed that $k>0$. Thus we have that $H^{k}\left(G / A, A^{H}\right)$ $=H^{k+d}\left(G / H, A^{H}\right)=0$. Since $G / H$ is cyclic and $d$ is odd, we obtain

Received by the editors September 1, 1966. 